\title{
The more things change, the more things stay the same: maternal attitudes 3 to 18 months postpartum
}

Katherine T. Cost (katherine.cost@utoronto.ca) ${ }^{1}$, André Plamondon ${ }^{2}$, Eva Unternaehrer ${ }^{3}$, Michael Meaney ${ }^{3}$, Meir Steiner ${ }^{4}$, Alison S. Fleming ${ }^{1}$, On behalf of the MAVAN team

1.Department of Psychology, University of Toronto Mississauga, Toronto, ON, Canada

2.Département des fondements et pratiques en éducation, Université Laval, Quebec, QC, Canada

3.Departments of Psychiatry and Neurology and Neurosurgery, Douglas Mental Health University Institute, McGill University, Montreal, QC, Canada

4.Departments of Psychiatry and Behavioral Neurosciences and Obstetrics and Gynecology, Women's Health Concerns Clinic, St. Joseph's Healthcare Hamilton, McMaster University, Hamilton, ON, Canada

\section{Keywords}

Maternal attitudes, Postpartum, Depression, Self-efficacy, Marital relationship

\section{Correspondence}

Katherine Tombeau Cost, Ph.D

Department of Psychology

University of Toronto Mississauga

252 Bloor Street West, Toronto, ON M5S 1V6,

Canada.

Tel: +905-569-4455

Fax: +905-569-4326

Email: katherine.cost@utoronto.ca

\begin{abstract}
Aim: Becoming a parent precipitates changes in new mothers' psychological and social domains. Previous literature has focused exclusively on pregnancy and the early postpartum, but parenting is an evolving process, necessitating adaption to changing circumstances. We extended previous literature and investigate the changes in the postpartum from 3 to 18 months that occur in maternal attitudes.
\end{abstract}

Methods: Using the Childbearing Attitudes Questionnaire, we collected data on mothers' ratings of maternal worries, self-efficacy, mother-infant bonding, relationship with the partner and interest in sex ( $n=171$ women). Data were analysed with a latent growth curve.

Results: Results demonstrated stability in all maternal attitudes after 3 months postpartum. Further, different maternal attitudes are affected by different variables. Maternal worries and self-efficacy are associated with parity, postpartum depression and child temperament. Interestingly, a negative evaluation of the relationship with the partner was only associated with breastfeeding status, while interest in sex was associated with parity, socio-economic status (SES) and depressive symptoms.

Conclusion: Despite general stability, different maternal attitudes related to different sets of variables. These patterns of attitudes in relation to relevant variables are discussed in terms of the literature on self-efficacy and gender roles, with important implications for clinical interventions.

\section{INTRODUCTION}

The transition to parenthood involves changes in mothers' behaviours, relationships, cognitions and attitudes. Past research has demonstrated that attitudes towards infants in general, social relationships with significant others, and self-concept change over the course of pregnancy and during the first postpartum months (1-5). Further, changes in attitudes have been related to other factors such as mothers' prior experience with child care, maternal depression and child temperament. Not surprisingly, different maternal attitudes show different patterns of changes across pregnancy and the postpartum period (2) and are related to different sets of variables. Here, we explored the greater

\begin{tabular}{l}
\hline Abbreviations \\
CAQ, Childbearing Attitudes Questionnaire; CTQ, Childhood \\
Trauma Questionnaire; EPDS, Edinburgh Postnatal Depression \\
Scale; IBQ, Infant Behaviour Questionnaire; MAVAN, Maternal \\
adversity, vulnerability and neurodevelopment; PBI, Parental \\
bonding instrument; SES, Socio-economic status.
\end{tabular}

trajectory of and influences on maternal attitudes and partner relationship, picking up where previous research has left off, at 3 months postpartum, through to 18 months postpartum.

\section{Key notes}

- From 3 to 18 months postpartum, maternal attitudes about self-efficacy, worries, mother-infant bonding, relationship with partner and interest in sex are stable, but are associated with different sets of intercept covariates, with important implications for clinical interventions and an inclusive approach to maternal wellness.

- Depressive symptoms, among other covariates, are associated with self-efficacy, worries, mother-infant bonding and interest in sex.

- Relationship with partner is negatively associated with breastfeeding. 
Generally, maternal attitudes about self-efficacy and feelings for children increase during the transition to motherhood. For instance, the attitudinal measure of maternal self-efficacy shows a robust increase from pregnancy to 3 months postpartum $(1,3)$. High maternal selfefficacy can also be linked to more positive postpartum mood, lower levels of difficult temperament in the child and by prior experience of caring for children (1,2,5-7). Another attitude that changes over the course of pregnancy is maternal worries, which includes mothers' concerns about childcare, their maternal competence and infants' health. Maternal worries decrease from the first trimester to 6 and 12 weeks postpartum as mothers gain experience $(2,3)$. How attached mothers feel to their offspring also increases over the course of pregnancy and into the postpartum (2). In one study, mother-foetus/infant bonding was positively related to higher postpartum mood, but was negatively associated with ambivalence about pregnancy and with previous experience with children (1). Overall, maternal attitudes about the maternal role and feelings about children improved over the course of pregnancy and the early postpartum period with an established effect of mood.

Previous studies have also demonstrated changes in mothers' perception of the quality of their relationship to their partners from pregnancy onward. Women reported more positive feelings about their partners during pregnancy than either before or after pregnancy (2). In fact, feelings about the partner relationship at 3 months postpartum appeared to be comparable to feelings about the partner relationship before pregnancy (2). The declines in assessment of the partner relationship have been attributed to the discrepancy between mothers' expectations during pregnancy of fathers' role in childcare and the amount of actual childcare fathers take on after the baby is born, during the postpartum (4,7). Apart from breastfeeding, a multicountry study finds that mothers consistently spend more than twice as much time engaged in childcare activities than fathers, regardless of the employment status of either parent or the age of the child (8). The discrepancy between expectations and reality based on breastfeeding as a component of childcare may decrease the perceived quality of the partner relationship. Another aspect of the partner relationship that decreases in quality during this time is associated with the mother's sex life. Indeed, mothers reported less interest in sex at 3 months postpartum compared to during pregnancy (3). Thus, women have a higher appraisal of the partner relationship during pregnancy than either before or immediately after pregnancy, which is influenced by mothers' expectations surrounding childcare and division of labour (4).

In sum, different maternal attitudes show different patterns of change across this important period in a woman's life. Maternal attitudes related to the child tend to become more positive across pregnancy and the early postpartum period and can be affected by a variety of factors related to the mother or the child. In contrast, maternal attitudes related to the partner tend to become more negative and seem to be related to expectations about division of household labour and childcare duties.

\section{Theoretical framework}

Within the framework of Belsky's (9) determinants of parenting, including three domains (1) personal and psychological resources (2), characteristics of the child and (3) contextual sources of stress and support (9), attitudes are encompassed in a mother's personal and psychological resources. According to this theory, of the three systems interacting as the determinants of parenting, personal psychological characteristics of the parent are paramount, should problems occur in the other two systems. That is to say, given optimal parental psychological characteristics, difficulties such as child temperament or suboptimal social conditions can be overcome or at least compensated to produce competent offspring. Furthermore, the personal and psychological characteristics of the mother can be influenced by her own upbringing, child temperament and contextual sources of stress and support, such as income and the partner relationship (9). Using this framework, with the conceptualisation of interaction between the three domains, we examined sets of factors associated with mothers' attitudes at the initial 3 months postpartum time point as well as at subsequent time points across the postpartum period, specifically at 6 and 18 months. To address factors related to personal and psychological resources, we included maternal mood, maternal early experiences and maternal age. To address factors related to characteristics of the child, we included child gender and temperament. To address factors related to contextual stress and support, we included socioeconomic status (SES; a combined measure of mother education and income), parity and breastfeeding.

\section{The current study}

While several studies have reported attitudinal changes during pregnancy and the early postpartum period, very few have addressed the changes in attitudes and relationships that occur in parallel with the dramatic development of the child in physical, motor, sensory, cognitive and social domains over the first 18 months postpartum (beyond 3 months postpartum) $(5,7,10)$. To fill this gap, this study tracks changes in maternal attitudes from three to eighteen months postpartum using the Childbearing Attitudes Questionnaire (CAQ). Included among the maternal attitudes studied are those whose pattern of change across pregnancy is well established, reflecting maternal worries, maternal self-efficacy and mother-infant bonding. We were also interested to know whether or how mothers' relationships with her partner may change, assessed in relationship with the partner and interest in sex. We predicted that a mother's assessment of her maternal self-efficacy and mother-infant bonding would increase, while maternal worries would decrease, related to the mother's increased experience and familiarity with the child and childcare and that these factors would be related to mood and infant characteristics. We predicted that relationship with partner and 
interest in sex would increase as the routine of childcare was negotiated and that these factors would be predicted by mood, breastfeeding status and parity.

\section{METHODS}

Participants

Participants were part of the Maternal Adversity, Vulnerability And Neurodevelopment (MAVAN) study, which longitudinally follows two cohorts of mothers and children in Montreal (Quebec) and Hamilton (Ontario) in Canada. The data in these analyses were restricted to the Hamilton sample. The MAVAN sample consisted of women who had been recruited during the second trimester of pregnancy (weeks 12-24) from a maternity hospital in Hamilton. All mothers in this sample were 18 years or older, first time enrolled in the study, singleton pregnancy, fluent in English and recruited between June 2004 and July 2014. Women with severe chronic illness that required ongoing care (other than hypertension, asthma or diabetes) were excluded from the study. Further, women with placenta previa, history of incompetent cervix, impending delivery or a foetus/infant affected by a major anomaly were also excluded from the study. Also, several women in treatment for depression and anxiety were recruited through a mental health clinic. Women who reported a history of bipolar disorder, psychosis or who reported they were suicidal or homicidal were excluded from the study. The Hamilton cohort consisted of 253 mothers on their first enrolment in the study. To have a comparable sample, basic inclusion criteria were to have one child at term. Ten participants were excluded because they did not meet these basic inclusion criteria (birth of multiples, termination of pregnancy, premature birth and still birth). A further 44 participants did not complete the 3-month visit. Of the remaining 177 mothers, 55 did not complete all of the questions in the $\mathrm{CAQ}$, resulting in different sample sizes for the analyses. Only participants who completed all questions related to a factor at the 3-month time point (the intercept) were included in the analysis of that factor. Participants who were excluded due to basic inclusion criterion and due to failure to complete the 3-month CAQ had significantly higher scores on the Edinburgh Postnatal Depression Scale (EPDS) at 3 months $(\mathrm{p}=0.030$, mean difference $=$ 1.954), had significantly higher scores on the Childhood Trauma Questionnaire (CTQ) $(\mathrm{p}=0.008$, mean difference 0.362 ), reported lower parental bonding instrument (PBI) maternal care $(p=0.001$, mean difference $=-6.178)$ and were significantly younger $(p=0.015$, mean difference $=-1.995)$, but were not significantly different from the included group in PBI father care, Infant Behaviour Questionnaire (IBQ) measures, EPDS at 6 or 18 months, breastfeeding at 3 or 6 months, SES or parity (See Table 1 for sample characteristics).

All aspects of the study had ethical approval from St. Joseph's Healthcare and McMaster University in Hamilton $\mathrm{ON}$, the University of Toronto in Toronto ON and all other study sites. All participants provided informed consent for their participation and assent for their infants' participation.

\begin{tabular}{lll} 
Table 1 Sample characteristics & $n$ & Mean (SE) \\
\hline Age of mother at time at delivery & 164 & $30.93(0.36)$ \\
EPDS at 3 months & 163 & $6.86(0.42)$ \\
EPDS at 6 months & 168 & $6.44(0.38)$ \\
EPDS at 18 months & 145 & $6.90(0.43)$ \\
CTQ & 171 & $-0.06(0.06)$ \\
PBI Mother Care & 155 & $27.54(0.62)$ \\
PBI Father Care & 154 & $22.21(0.84)$ \\
IBQ Surgency Extraversion & 162 & $4.20(0.06)$ \\
IBQ Negative Affectivity & 168 & $3.02(0.05)$ \\
IBQ Orienting Regulation & 168 & $5.12(0.05)$ \\
& $n$ & $\%$ \\
\hline \% Breastfeeding at 3 months & 162 & 75 \\
\% Breastfeeding at 6 months & 161 & 62 \\
$\%$ Breastfeeding at 12 months & 164 & 30 \\
$\%$ Primiparous & 160 & 43 \\
\% High education, high income & 154 & 82 \\
\hline
\end{tabular}

EPDS, Edinburgh Postnatal Depression Scale; CTQ, Childhood Trauma Questionnaire; PBI, Parental bonding instrument; IBQ, Infant Behaviour Questionnaire.

\section{Procedure}

Maternal attitudes

The CAQ was given to mothers at 3 months, 6 months and 18 months postpartum to assess maternal attitudes on aspects of pregnancy, caregiving and relationships. The CAQ includes 83 items, with a 7-point Likert scale response from disagree strongly (1) to agree strongly (7). Factors from an earlier version of the CAQ (with seven fewer items) have been previously established with high reliability: maternal worries, maternal self-efficacy, mother-infant bonding, relationship with partner and interest in sex $(1,3)$. Each factor includes 3-7 questions, counter-balanced for direction to reduce response bias. We used in our analyses these previously established factors (3).

\section{Edinburgh postnatal depression scale}

The EPDS is a questionnaire with 10 items to screen for postpartum depression. The EPDS has high sensitivity $(86 \%)$ and high specificity $(78 \%)(11)$. Participants completed the EPDS at 3, 6 and 18 months postpartum. As we know that postpartum depression is dynamic, we conducted a repeated-measures analysis on all three EPDS scores of our sample and found that EDPS was both low and stable (linear $F(1,132)=0.046, p=0.830$; quadratic $F(1,132)=2.279, p=0.134$; see Table 1$)$.

\section{Infant temperament}

Infant temperament was assessed at 3 months of age with the IBQ, designed to assess infant temperament between 3 months and 12 months. The IBQ consists of 184 questions encompassing 14 subscales asking mothers to rate their infants' behaviour on several items that comprise the scales in the past week on a 7-point Likert scale from 'never' to 'always'. From these questions, three broad 
factors are constructed: surgency/extroversion, negative affectivity and orienting/regulation. The IBQ is well established with high internal consistency for the three super factors (0.91-0.93) and inter-rater reliability between mothers and secondary caregivers for the three super factors (0.31-0.70) (12).

\section{Parental bonding instrument}

The PBI was used to retrospectively assess the quality of care and degree of over-protection or control mothers received from their own parents. This self-report questionnaire includes 50 items, 25 for each parent, resulting in four factors: maternal care, maternal over-protection, paternal care and paternal over-protection. The PBI is a wellresearched and stable construct, with high internal consistency $(0.74-0.95)$ and test-retest reliability $(0.74-0.94)$ in a community sample (13).

\section{Childhood trauma questionnaire}

The CTQ was used to retrospectively assess experiences of childhood abuse, neglect and trauma in participants at the visit during 12-18 weeks of pregnancy. This self-report questionnaire consists of 28 items to assess five types of abuse: physical, emotional and sexual abuse; emotional and physical neglect. The CTQ has high reliability for five factors (0.79-0.94), high test-retest (0.88) and high concurrent validity with an external measure of childhood trauma $(\mathrm{r}=0.25-0.58)(14)$.

\section{Breastfeeding}

Breastfeeding data were derived from the Health and WellBeing of Mothers and their Newborns Questionnaire, completed at 3 months postpartum. Mothers indicated whether they were breastfeeding exclusively, partially or not at all. All mothers indicated that they were breastfeeding and not expressing milk. From these questions, we created a dichotomised variable: any breastfeeding at 3 months or no breastfeeding at 3 months.

Age of mother at delivery, parity, socio-economic status and child gender

Demographic characteristics, such as age at delivery, parity and socio-economic status, were collected during the second trimester of pregnancy for the mother and at 3 months postpartum for the infant gender with the Health and Well-Being of Mothers and their Newborns Questionnaire. The SES variable was calculated to include both income and education, with three groups represented, based on national census data (15): 1 = low income, low education; 2 = low income and high education OR high income, low education; 3 = high income, high education. Based on national census data, low income was defined as less than \$21358 total family income after tax, while high income is defined as more than \$21 359 total family income after tax. Low education is defined as high school graduation or less (16). Our SES groups were defined by the amount of socioeconomic risk that has previously been associated with parenting outcomes (17), with group 1 having risks associated with both income and education, group 2 have risks associated with either income or education, and group 3 have no risks associated with income or education.

\section{Analysis}

CAQ factor cohesion with Cronbach's alpha

The CAQ factors have been previously analysed for cohesion using Cronbach's alpha during pregnancy and at 3 months postpartum (3). We have reanalysed the factors with this sample at each of the time points $(3,6$, and 18 months postpartum) using Cronbach's alpha. In our study data, we found that several factors in the CAQ have internal consistency across the postpartum period from 3 to 18 months. Internal consistency for maternal worries (0.638-0.677), maternal self-efficacy (0.693-0.717) and interest in sex (0.639-0.652) fell within the acceptable range. Relationship with partner $(0.818-0.820)$ has high internal consistency. Bonding had a lower internal consistency (0.531-0.631). Despite the lower consistency for this factor, we chose to retain the bonding factor given the strong effects of time on change in the bonding factor in previous work (2).

\section{Correlations with factors by variables}

A survey of the literature on maternal attitudes and the transition to motherhood identifies several possible variables that could affect maternal attitudes at 3 months postpartum (the intercept) and also the change over time of maternal attitudes from 3 to 6 to 18 months postpartum (the slope). Many of these variables are available in the MAVAN data set. However, due to limitations of sample size and the problem of multicollinearity, we needed to restrict the number of variables included in the conditional latent growth curve model. To determine which variables to include in each factor model, we first produced a correlation matrix using SPSS (IBM, Inc., Armonk, NY, USA) between each CAQ factor at each time point with several candidate variables supported by past research, such as SES, mother's age at birth, child gender, parity, IBQ, PBI, CTQ, EPDS and breastfeeding. Any variable that was found to be significantly correlated with the CAQ factor at any of the three time points was included in the conditional latent growth curve model for that variable (see Supplemental Materials, Table S1).

\section{Imputation of missing data}

We ran 50 imputations for all missing data in the CAQ and covariates using Mplus (Muthén and Muthén, Los Angeles, CA, USA). To be included in the imputations, participants had to have completed the 3-month assessment of the CAQ.

\section{Latent growth curve}

To assess change over time in maternal attitudes, we constructed latent growth curve models in Mplus (Muthén and Muthén, Los Angeles, CA, USA). We first ran unconditional latent growth curve models, that is, including the CAQ factor at the three time points without covariates. The 
unconditional model allows us to create the model with the best fit for the data based on the chi-squared test statistic and fit indices (18). In the unconditional model, we are concerned with the goodness of fit, information about group averages in the start point (intercept mean) and trajectory (slope mean), and evidence of interindividual differences in the start point (intercept variance) and trajectory (slope variance) (19).

We then ran conditional latent growth curve models, that is models which included the CAQ factor at all three time points as well as any variables that were significantly correlated with the factor at any time point, as previously described. The conditional model includes predictors of growth or change over time. Our conditional models include only time-invariant covariates, which do not change in value over time because they are either attributes of the individual or have only been measured at one time point, either prior to 3 months postpartum or at 3 months postpartum (the intercept). The inclusion of covariates allows us to test whether these individual characteristics predict the interindividual differences in either the initial level of the attitude at 3 months postpartum (the intercept) or how that attitude may change over time from 3 months postpartum to 18 months postpartum (the slope) (18).

All models had the slope constrained at 0 (3 months), 0.2 (6 months), and 1.0 (18 months) to reflect the span of 3-18 months with the rate of change tracking the actual amount of time between measurements, and the intercept at 3 months (0). Further, to improve model fit, residual variances of the variables included in the growth models were constrained in all models. The fit indices indicate that the data are consistent with the specified model in that the model reproduces the variance-covariance matrix. Models were chosen based on fit indices, including $\chi^{2}$, RMSEA (values $<0.05$ indicate a good fit), CFI/TLI (values $>0.95$ indicate a good fit) and SRMR (values $<0.05$ indicate a good fit) (19).

\section{RESULTS}

\section{Correlations with factors by variables}

We found several variables to include in the conditional model for each factor. Only those variables that were significantly correlated with the factor at any of the three time points $(3 \mathrm{~m}, 6 \mathrm{~m}$ and $18 \mathrm{~m})$ were included in the imputation and conditional model for the factor. Three variables, mother's age, child gender and PBI mother overprotection, were not significantly correlated with any of the factors at any time point and were therefore not included in any of the conditional models.

All model fit indices are summarised in Table 2. All findings are summarised in Table 3. Only significant results will be discussed.

\section{Maternal worries, maternal self-efficacy and mother- infant bonding \\ Model fit indices}

In the unconditional and conditional models for maternal worries and maternal self-efficacy, the model fits were adequate. For mother-infant bonding, unconditional model fit indices were good and the conditional model fit indices were adequate.

\section{Conditional model}

In the conditional model, the mean at 3 months for maternal worries, maternal self-efficacy and mother-infant bonding was significantly greater than $0 \quad(p=0.002$, $\mathrm{p}<0.0005, \mathrm{p}<0.0005)$, indicating that mothers rated their worries, self-efficacy and bonding as higher than 'not at all like' themselves (one on the Likert scale). The variance at 3 months postpartum was significant in all three factors (maternal worries, maternal self-efficacy and mother-infant bonding), indicating significant individual differences at 3 months postpartum $(\mathrm{p}<0.0005, \mathrm{p}<0.0005, \mathrm{p}=0.001)$. Significant associations with the mean at 3 months of maternal worries were IBQ negative affectivity $(p=0.031)$ with mothers who rated their infants as higher on negative affectivity expressing higher maternal worries, EPDS at 3 months postpartum $(\mathrm{p}<0.0005)$ with more depressed mothers expressing higher maternal worries and parity $(\mathrm{p}<0.0005)$ with first time mothers expressing higher maternal worries (Table 3). Significant associations with the mean at 3 months of maternal self-efficacy were EPDS at 3 months postpartum $(\mathrm{p}<0.0005)$ with more depressed mothers expressing lower feelings of maternal self-efficacy and parity $(\mathrm{p}<0.0005)$ with multiparous mothers expressing higher feelings of maternal self-efficacy (Table 3). Significant associationss for mother-infant bonding (at 3 months) were EPDS at 3 months postpartum

\begin{tabular}{|c|c|c|c|c|c|c|c|c|c|c|}
\hline \multirow[b]{2}{*}{ Model } & \multicolumn{2}{|c|}{$\begin{array}{l}\text { Maternal worries } \\
(n=171)\end{array}$} & \multicolumn{2}{|c|}{$\begin{array}{l}\text { Maternal self-efficacy } \\
(n=169)\end{array}$} & \multicolumn{2}{|c|}{$\begin{array}{l}\text { Mother-infant bonding } \\
(n=171)\end{array}$} & \multicolumn{2}{|c|}{$\begin{array}{l}\text { Relationship with partner } \\
(n=155)\end{array}$} & \multicolumn{2}{|c|}{$\begin{array}{l}\text { Interest in sex } \\
(n=169)\end{array}$} \\
\hline & U & $\mathrm{C}$ & U & $\mathrm{C}$ & $\mathrm{U}$ & $C$ & U & $\mathrm{C}$ & U & $\mathrm{C}$ \\
\hline$\chi^{2}$ & 4.164 & 18.200 & 4.242 & 10.381 & 0.679 & 22.696 & 2.671 & 8.033 & 6.296 & 11.212 \\
\hline $\mathrm{CFI}$ & 0.975 & 0.960 & 0.980 & 0.992 & 1.000 & 0.951 & 0.986 & 0.997 & 0.958 & 0.981 \\
\hline TLI & 0.968 & 0.903 & 0.981 & 0.994 & 1.043 & 0.945 & 1.003 & 1.047 & 0.958 & 0.962 \\
\hline RMSEA & 0.063 & 0.058 & 0.041 & 0.019 & 0.001 & 0.035 & 0.020 & 0.006 & 0.074 & 0.032 \\
\hline SRMR & 0.043 & 0.022 & 0.158 & 0.061 & 0.092 & 0.137 & 0.057 & 0.026 & 0.053 & 0.026 \\
\hline
\end{tabular}

$\mathrm{U}$, unconditional model with no predictors/covariates included; C, conditional models with correlated predictors/covariates included. 


\begin{tabular}{|c|c|c|c|c|c|}
\hline & Maternal worries & Maternal self-efficacy & Mother-infant bonding & Relationship with partner & Interest in sex \\
\hline \multicolumn{6}{|l|}{ Intercept on } \\
\hline $\mathrm{PBI}$ mother care & $-0.015(0.121)$ & N.I. & $0.005(0.006)$ & $0.218(0.126)$ & $-0.065(0.124)$ \\
\hline PBI father care & $-0.135(0.101)$ & N.I. & N.I. & $-0.031(0.117)$ & N.I. \\
\hline PBI father over-protection & N.I. & $-0.034(0.087)$ & $-0.008(0.006)$ & N.I. & N.I. \\
\hline CTQ (mean z-score) & $0.084(0.112)$ & $-0.049(0.077)$ & N.I. & N.I. & $-0.076(0.114)$ \\
\hline $\mathrm{IBQ}$ surgency/extroversion & $-0.136(0.114)$ & $-0.034(0.106)$ & N.I. & $0.138(0.133)$ & N.I. \\
\hline IBQ negative affectivity & $0.210(0.099)^{*}$ & $-0.118(0.092)$ & $-0.133(0.078)$ & N.I. & $-0.084(0.089)$ \\
\hline IBQ orienting/self-regulation & $0.017(0.126)$ & $0.177(0.107)$ & $0.020(0.094)$ & $0.090(0.118)$ & N.I. \\
\hline EPDS (3 months postpartum) & $0.503(0.095)^{*}$ & $-0.517(0.097)^{*}$ & $-0.036(0.013)^{*}$ & $-0.171(0.099)$ & $-0.416(0.084)^{*}$ \\
\hline Parity $(0=$ primi, $1=$ multi $)$ & $-0.620(0.162)^{*}$ & $0.300(0.075)^{*}$ & N.I. & $-0.179(0.187)$ & $-0.458(0.175)^{*}$ \\
\hline SES $(1=$ low, $3=$ high $)$ & N.I. & N.I. & $-0.275(0.093)^{*}$ & N.I. & $-0.182(0.089)^{*}$ \\
\hline Any breastfeeding $(0=$ no, $1=$ yes $)$ & N.I. & N.I. & N.I. & $-0.485(0.202)^{*}$ & N.I. \\
\hline \multicolumn{6}{|l|}{ Slope on } \\
\hline $\mathrm{PBI}$ mother care & $-0.023(0.279)$ & N.I. & N.I. & $-0.099(0.262)$ & $0.168(0.292)$ \\
\hline PBI father care & $0.076(0.223)$ & N.I. & N.I. & $0.090(0.241)$ & N.I. \\
\hline PBI father over-protection & N.I. & $0.000(0.002)$ & N.I. & N.I. & N.I. \\
\hline CTQ (mean z-score) & $0.162(0.346)$ & $-0.098(0.161)$ & N.I. & N.I. & $-0.074(0.279)$ \\
\hline IBQ surgency/extroversion & $-0.180(0.301)$ & $0.424(0.260)$ & N.I. & $-0.302(0.375)$ & N.I. \\
\hline IBQ negative affectivity & $-0.241(0.296)$ & $0.027(0.188)$ & N.I. & N.I. & $-0.044(0.209)$ \\
\hline $\mathrm{IBQ}$ orienting/self-regulation & $0.165(0.277)$ & $-0.621(0.337)$ & N.I. & $0.200(0.287)$ & N.I. \\
\hline EPDS (3 months postpartum) & $-0.279(0.298)$ & $0.192(0.210)$ & N.I. & $0.046(0.193)$ & $0.406(0.387)$ \\
\hline Parity $(0=$ primi, $1=$ multi $)$ & $0.786(0.830)$ & $-0.180(0.141)$ & N.I. & $0.136(0.360)$ & $1.062(1.123)$ \\
\hline SES $(1=$ low, $3=$ high $)$ & N.I. & N.I. & N.I. & N.I. & $0.185(0.300)$ \\
\hline Any breastfeeding $(0=$ no, $1=$ yes $)$ & N.I. & N.I. & N.I. & $0.232(0.493)$ & N.I. \\
\hline \multicolumn{6}{|l|}{ Means/intercept } \\
\hline Intercept & $2.868(0.996)^{*}$ & $5.003(0.767)^{*}$ & $7.722(0.829)^{*}$ & $3.153(0.942)^{*}$ & $6.141(0.975)^{*}$ \\
\hline Slope & $-0.081(1.280)$ & $1.512(1.247)$ & N.I. & $0.065(1.194)$ & $-1.443(1.312)$ \\
\hline \multicolumn{6}{|l|}{ Variances } \\
\hline Intercept & $0.391(0.097)^{*}$ & $0.384(0.094)^{*}$ & $0.158(0.047)^{*}$ & $0.853(0.170)^{*}$ & $0.906(0.182)^{*}$ \\
\hline Slope & $0.256(0.253)$ & $0.292(0.194)$ & N.I. & $0.491(0.382)$ & $0.290(0.391)$ \\
\hline Intercept-slope correlation & $0.006(0.104)$ & $-0.101(0.093)$ & N.I. & $-0.356(0.204)$ & $-0.177(0.195)$ \\
\hline
\end{tabular}

N.I., predictor Not Included in model; CTQ, Childhood Trauma Questionnaire; EPDS, Edinburgh Postnatal Depression Scale; SCS, socio-economic status, PBI, Parental bonding instrument.

*p $<0.05$.

Significant predictors are in bold to improve readability.

$(\mathrm{p}=0.001)$ with more depressed mothers expressing less bonding to their infants and SES $(p=0.002)$ with lower SES mothers expressing more bonding to their infants (Table 3; Fig. 1A).

\section{Relationship with partner and interest in sex \\ Model fit indices}

In the unconditional and conditional models for relationship with partner, the model fits were very good. The unconditional and conditional models for interest in sex had adequate model fit indices.

\section{Conditional model}

In the conditional model, the means at 3 months for both relationship with partner and interest in sex were significant $(p=0.001 ; p<0.0005)$. The variances of attitudes on relationship with partner and interest in sex at 3 months were significant for both factors, indicating significant individual differences at 3 months postpartum $(\mathrm{p}<0.0005 ; \mathrm{p}<0.0005)$. For relationship with partner, the only significant association with the mean at 3 months was any breastfeeding at 3 months $(\mathrm{p}=0.016)$, with breastfeeding mothers indicating less satisfaction with the relationship with the partner. For interest in sex, significant associations with the mean at 3 months included SES $(\mathrm{p}=0.040)$, with women with lower SES endorsing higher interest in sex, EPDS at 3 months postpartum $(\mathrm{p}<0.0005)$, with more depressed women indicating less interest in sex and parity $(\mathrm{p}=0.009)$, with multiparous women indicating less interest in sex (Table 3; Fig. 1B).

\section{DISCUSSION}

Overall, our results indicate remarkable stability in all maternal attitudes at 3 to 18 months postpartum. We also see that different maternal attitudes are affected by different variables. Maternal worries are lower in multiparous women compared to primiparous women. However, maternal worries are higher in mothers with higher levels of depression and mothers whose infants have high negative 

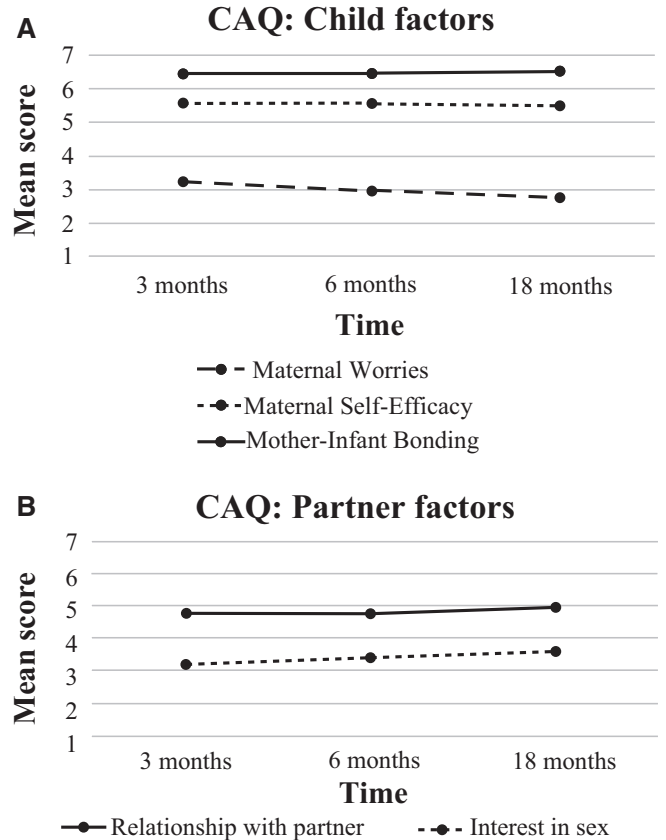

Figure 1 Childbearing Attitudes Questionnaire (CAQ) factors over 3 18 months postpartum. Means are obtained from the unconditional model. (A) Child-related factors, (B) Partner-related factors.

affectivity. In contrast, maternal self-efficacy is increased in multiparous mothers compared to primiparous mothers, but decreased in women with higher levels of depression. With regard to the relationship with the partner, breastfeeding was the only association, significantly decreasing the assessed quality of the relationship with the partner. Interest in sex was negatively related to higher levels of depression. Multiparous women also had lower interest in sex. Also, lower SES associated with higher interest in sex.

The pattern of findings in maternal attitudes towards the child indicates that higher levels of depressive symptoms at 3 months postpartum related to maternal worries and selfefficacy, even 15 months later. Higher depression and anxiety has previously been related to lower scores on maternal attitudes such as maternal responsibility and role idealisation (5). Low maternal self-efficacy has been found to predict the stability of depression at 3 months postpartum (20). Another study found an association between lower self-efficacy and lower mood at 1 year postpartum (6). Previous research has also found that treatment to alleviate the symptoms of postpartum depression is insufficient to ameliorate the mother-infant relationship (21). While treatment for postpartum depression decreased parenting stress, which does include questions on maternal role competence, parenting stress was still higher in depressed mothers than in nondepressed mothers, indicating that the treatment did not specifically or indirectly improve feelings of maternal self-efficacy. Further, low maternal confidence has been found to be related to increased rumination in depressed mothers (22). Moreover, the increased rumination in depressed mothers also impaired problem-solving, providing a link between low maternal self-efficacy and actual impaired parenting ability (22). Previous research has also found that self-efficacy is decreased in postpartum mothers with low levels of social support and that these two factors combine to predict postpartum depression (23). Our results are concordant with this literature, finding that depression symptoms at 3 months related to outcomes 15 months later, further indicating that depression at 3 months is a relevant measure to identify mothers that are more likely to present lower maternal self-efficacy and higher maternal worries 15 months later.

With regard to the relationship with the partner, we were most interested in breastfeeding associating with a more negative relationship with the partner. Previous research has demonstrated that there is a strong negative relationship between the quality of the partner relationship and the amount of household chores carried out by the female partner after the birth of a child (24). Because breastfeeding is necessarily carried out by the mother, it may be creating an unequal division of labour, particularly if the father feels excluded or lacks confidence in care-giving (25). Expectations may also be involved in the postpartum decrease in mothers' feelings about their partners. Postpartum women have reported that their husbands did less housework than expected (4). Only $12 \%$ of postpartum women reported an equal sharing of household chores with their partners (4). With regard to childcare, $41 \%$ of new mothers reported doing much more childcare than their partners (4). Depending upon the involvement of the father in other aspects of childcare, breastfeeding may create an inherently unequal division of labour, which gives rise to a mother's negative appraisal of the relationship with her partner. A previous study found that a coparenting intervention that included breastfeeding support by the partner increased maternal satisfaction with her partner's involvement and assistance (25). In contrast to previous studies, which have found an association between depression symptoms and relationship with partner $(7,10)$, we found no association between depressive symptoms relationship with partner; however, higher depressive symptoms were associated with lower interest in sex, which may be a reflection of and/or an influence on the relationship with the partner. The associations between breastfeeding status, depressive symptoms, relationship with partner and interest in sex merit further study.

Although previous studies have found changes in maternal attitudes during pregnancy and up to 3 months postpartum, as our study focuses exclusively on 3-18 months postpartum, we find that maternal attitudes about the child and the partner are stable from 3 to 18 months postpartum. Future studies could consider the timing of interventions and psycho-educational strategies prior to 3 months postpartum to increase the likelihood of improving maternal attitudes and maternal well-being.

\section{Limitations}

As this study was conducted in a longitudinal manner, there are some limitations inherent to longitudinal data collec- 
tion, such as sample biasing due to drop outs, conditioning and seam bias. Here, we attempt to control for drop out by using imputed data, but we still have a small sample relative to the number of analyses. Further, our analyses have shown little evidence of seam bias. Although conditioning to the questionnaire is not something we have been able to control, a previous study using this questionnaire in both a longitudinal sample and a cross-sectional sample have found similar results in both samples, indicating that the effect of repeated assessments by the same questionnaire is not a major source of error (2). Another potential limitation of this study is the sensitivity of the questions on the questionnaire and the marginal reliability of some of the factors created. It may be that questions address larger constructs or ongoing characteristics of the mother and her attitudes. Also, our inclusion of covariates was based on linear associations, but some covariates may have quadratic associations. Finally, participants excluded for failure to complete the CAQ instrument did have higher scores on depression and childhood trauma and lower scores on the early experience of maternal care and were younger than those who completed the instrument and were included in the analysis. While this reduces the generalisability of the outcomes, of these covariates, depression scores were significantly associated with multiple outcomes in our study and these associations may have been strengthened in the full sample.

\section{CONFLICT OF INTEREST}

No potential conflict of interest was reported by the authors.

\section{References}

1. Fleming AS, Ruble DN, Flett GL, Shaul DL. Postpartum adjustment in first-time mothers: relations between mood, maternal attitudes, and mother-infant interactions. Dev Psychol 1988; 24: 71-81.

2. Fleming AS, Ruble D, Krieger H, Wong PY. Hormonal and experiential correlates of maternal responsiveness during pregnancy and the puerperium in human mothers. Horm Behav 1997; 31: 145-58.

3. Ruble DN, Brooks-Gunn J, Fleming AS, Fitzmaurice G, Stangor C, Deutsch F. Transition to motherhood and the self: measurement, stability, and change. J Pers Soc Psychol 1990; 58: 450-63.

4. Ruble DN, Fleming AS, Hackel LS, Stangor C. Changes in the marital relationship during the transition to first time motherhood: effects of violated expectations concerning division of household labor. J Pers Soc Psychol 1988; 55: 78-87.

5. Sockol LE, Epperson CN, Barber JP. The relationship between maternal attitudes and symptoms of depression and anxiety among pregnant and postpartum first-time mothers. Arch Womens Ment Health 2014; 17: 199-212.

6. Horwitz SM, Briggs-Gowan MJ, Storfer-Isser A, Carter AS. Prevalence, correlates, and persistence of maternal depression. J Women's Health 2007; 16: 678-91.

7. Sayil M, Gure A, Ucanok Z. First time mothers' anxiety and depressive symptoms across the transition to motherhood: associations with maternal and environmental characteristics. Women Health 2006; 44: 61-77.

8. Craig L, Mullan K. How mothers fathers share childcare: a cross-national time-use comparison. Am Sociol Rev, 2011; 76: 834-61.

9. Belsky J. The determinants of parenting: a process model. Child Dev 1984; 55: 83-96.

10. Mortensen O, Torsheim T, Melkevik O, Thuen F. Adding a baby to the equation. Married and cohabiting women's relationship satisfaction in the transition to parenthood. Fam Process 2012; 51: 122-39.

11. Cox JL, Holden JM, Sagovsky R. Detection of postnatal depression. Development of the 10-item Edinburgh Postnatal Depression Scale. Br J Psychiatry 1987; 150: 782-6.

12. Gartstein MA, Rothbart MK. Studying infant temperament via the Revised Infant Behavior Questionnaire. Infant Behav Dev 2003; 26: 64-83.

13. Wilhelm K, Parker G. Reliability of the parental bonding instrument and intimate bond measure scales. Aust N Z J Psychiatry 1990; 24: 199-202.

14. Burgermeister D. Childhood adversity: a review of measurement instruments. J Nurs Meas 2007; 15: 163-76.

15. Statistics Canada. Income and earnings. 2008.

16. Canada S. Education indicators in Canada: an international perspective 2011. 2011. Available at: http://www.statcan.gc.ca/ pub/81-604-x/81-604-x2011001-eng.htm. (cited March 9, 2015).

17. Belsky J, Bell B, Bradley RH, Stallard N, Stewart-Brown S. Socioeconomic risk, parenting during the preschool years and child health age 6 years. Eur J Pub Health 2007; 17: 508-13.

18. Curran PJ, Obeidat K, Losardo D. Twelve frequently asked questions about growth curve modeling. J Cogn Dev 2010; 11: 121-36.

19. Byrne BM. Structural equation modeling with Mplus: basic concepts, applications, and programming. Multivariate applications series Harlow L, editor. 2012, New York, NY: Routledge: 412.

20. Abdollahi F, Zarghami M, Shariff-Ghazali S, Munn-Sann L. Stability of depressive symptoms over 3 months post-partum. Early Interv Psychiatry 2015. doi:10.1111/eip.12215.

21. Forman DR, O'Hara MW, Stuart S, Gorman LL, Larsen KE, Coy KC. Effective treatment for postpartum depression is not sufficient to improve the developing mother-child relationship. Dev Psychopathol 2007; 19: 585-602.

22. O'Mahen HA, Boyd A, Gashe C. Rumination decreases parental problem-solving effectiveness in dysphoric postnatal mothers. J Behav Ther Exp Psychiatry 2015; 47: 18-24.

23. Zhang Y, Jin S. The impact of social support on postpartum depression: the mediator role of self-efficacy. J Health Psychol, 2014; [Epub ahead of print].

24. Belsky J, Lang M, Huston TL. Sex typing and division of labor as determinants of marital change across the transition to parenthood. J Pers Soc Psychol 1986; 50: 517-22.

25. Abbass-Dick J, Stern SB, Nelson LE, Watson W, Dennis CL. Coparenting breastfeeding support and exclusive breastfeeding: a randomized controlled trial. Pediatrics 2015; 135: 102-10.

\section{SUPPORTING INFORMATION}

Additional Supporting Information may be found in the online version of this article:

Table S1 Correlations between CAQ factors at 3, 6, and 18 months with predictors and covariates. 